\title{
Computing Corpus Callosum as Biomarker for Degenerative Disorders
}

\author{
Thomas Kovac, Sammy Rogmans and Frank Van Reeth \\ Expertise Centre for Digital Media, Hasselt University - $t U L$ - iMinds, Wetenschapspark 2, 3590 Diepenbeek, Belgium
}

Keywords: Biomarker, Corpus Callosum, Image Registration, GPGPU, CUDA.

\begin{abstract}
The developed framework can automatically extract a plane with minimal corpus callosum area while simultaneously segmenting it. The method used, introduced by Ishaq, treats the corpus callosum area as a function of the plane extraction parameters and it uses deformable registration to generate a displacement field that can be used for the calculation of the corpus callosum area. Our registration framework is accelerated using CUDA, which enables researchers to benchmark huge amounts of data (patients) to test the hypothesis of the corpus callosum evolution as a biomarker for multiple degenerative disorders like e.g. Alzheimer disease and multiple sclerosis (MS).
\end{abstract}

\section{INTRODUCTION}

Multiple Sclerosis (MS) is an inflammatory disorder of the brain and spinal cord and it has been known to cause atrophy and deformation in the corpus callosum. Longitudinal studies try to quantify these changes by using medical image analysis techniques for measuring and analyzing the size and shape of the corpus callosum. These medical techniques mostly analyze and track changes in the corpus callosum by measuring the cross-sectional area by selecting a 2$\mathrm{D}$ measuring plane, typically the midsagittal plane. If this identification is done incorrectly, the measurement of the corpus callosum area will also be faulty. Therefore, an automation of finding a plane with minimal corpus callosum area is implemented to ensure that the measurement of the cross-sectional area is done correctly with high accuracy.

The employed method of finding a plane with minimal corpus callosum area depends heavily on deformable image registration. As the image registration process must be performed multiple times, it is important that the registration is performed as quickly, and correctly, as possible. The use of a GPU greatly improves computation time, so this framework is built out of algorithms and data structures that exploit its parallel computation capabilities and hardware. The implemented framework is algorithmically inspired by the work of various research groups and combines the advantageous approaches into one method.

\section{RELATED WORK}

There is a vast amount of literature devoted to image registration. In this section, we mention several dominant image registration approaches. We also consider related research in finding a plane with minimal corpus callosum area.

\subsection{Image Registration}

To date, there are multiple deformable registration algorithms proposed and validated. This includes thin-plate splines (Bookstein and Green, 1993), viscous fluid registration (Christensen et al., 1996), surface matching (Thompson and Toga, 1996), finiteelement models (Metaxas, 1997), spline-based registration (Szeliski and Coughlan, 1997), demons registration (Thirion, 1998), and B-spline registration (Rueckert et al., 1999).

Spline-based registration methods are currently very popular. Their flexibility and robustness provide the ability to perform mono-modal and multimodal registration. The appealing characteristics of both free-form deformation and spline-based methods are the most important reason why many studies have been conducted involving these techniques. Rueckert et al. present a method, using cubic B-splines curves to define a displacement field, which maps voxels in a moving image to those in a reference image (Rueckert et al., 1999). Each individual voxel movement between reference and moving image, is parameterized in terms of uniformly spaced control points that are

138 Kovac T., Rogmans S. and Van Reeth F. 


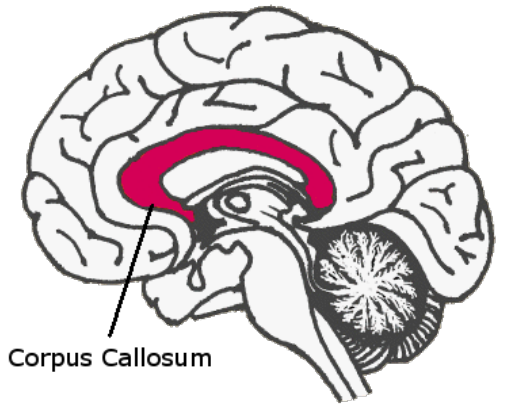

Figure 1: Corpus callosum cross-section.

aligned with the voxel grid. The displacement vectors are obtained via interpolation of the control point coefficients, using piecewise, continuous B-spline basis functions.

Besides the popularity of spline-based registration methods and their potential to greatly improve the geometric precision for a variety of medical procedures, they are usually computationally intensive (Shackleford et al., 2010). (Shackleford points to reports of algorithms requiring hours to compute for demanding image resolutions (Rohde et al., 2003; Aylward et al., 2007), depending on the specific algorithm implementation. To remedy these shortcomings, Shackleford has proposed a GPU-based image registration design to accelerate both the B-spline interpolation problem as well as the cost-function gradient computation that makes use of coalesced accesses to the GPU global memory and an efficient use of shared memory.

\subsection{Minimal Corpus Callosum Area}

The corpus callosum joins the two cerebral hemispheres. It acts as a bridge existing out of nerve fibers and it provides the exchange of information across the two hemispheres. Neurological diseases have been known to affect the shape and size of the anatomical structures in the brain. Measurement of this change and its correlation with disease progression has been one of the goals of clinical research (Hubel, 1995; Simon, 2006; Compston and Coles, 2008). See Figure 1 for a graphical representation of the cross-section of a corpus callosum.

MS often affects the brain ventricles width, overall brain width and specially the corpus callosum whose area loss has been documented in longitudinal studies (Simon et al., 2006; Simon, 2006). These effects on the corpus callosum size have generally been quantified by measuring the cross-sectional area of the corpus callosum. This was done through selecting a 2-D measuring plane from a MRI volume, typically the midsagittal plane, and measuring the area of the corpus callosum cross-section in this plane. This method is highly dependent on the accurate selection of the measuring plane, as it influences the measurement of the area. As longitudinal studies require patients to undergo several scans over a long period of time, factors such as the error in positioning of the human head in the MRI scanner between two scans can potentially be a source of error in the selection of the midsagittal plane and consequently the measurement of the corpus callosum area.

Another popular brain morphometry measure is the brain volume. However, work by Duning et al. has challenged the use of the whole brain volume as a measure of brain atrophy due to its susceptibility to dehydration and rehydration effects (Duning and Kloska, 2005), whereas the corpus callosum, being a dense fibrous structure, is hypothesized to be less sensitive to hydration effects and its area is potentially a more reliable measure of neuro-degeneration and atrophy (Ishaq, 2008).

\section{MOTIVATION}

In the previously mentioned studies, the changes in the corpus callosum size have been quantified by measuring the corpus callosum cross-sectional area imbedded in a measurement plane. Therefore, it is paramount that the accurate measurement of this change in corpus callosum area is dependent on the repeatable identification of the same corpus callosum cross-section in different scans. Typically, the midsagittal plane (MSP) serves as this measurement plane.

Ishaq emphasizes on two major disadvantages of using MSP as the plane for measurement of the corpus callosum area (Ishaq, 2008). First, accurate and repeatable identification of the same corpus callosum cross-section is difficult due to potential changes in brain anatomy over time, which can potentially affect the interhemispheric symmetry and the shape of the interhemispheric fissure. Even small errors in the selection of the MSP have been found to mystify the interpretation of the actual changes in the corpus callosum area due to pathology. Second, these extraction methods only incorporate the information regarding the brain hemispheric symmetry and the interhemispheric fissure, but completely ignore the characteristics of the corpus callosum itself. However, the rate of corpus callosum atrophy and deformation can be independent of the rate of the hemispheric degeneration, therefore the repeatable extraction of the same corpus callosum cross-section becomes difficult even 
for those cases where the brain hemispheres undergo minimal or no change between scans. These issues cast doubt on the reliability of employing the MSP as the measurement plane for measuring the corpus callosum area.

To this end, Ishaq proposed a novel and clinically meaningful criterion for defining an ideal measurement plane for the corpus callosum area measurement. It differs from symmetry and feature-based methods because it is based on finding the plane which optimizes certain physical properties of the corpus callosum itself. This is clinically more meaningful and specifically tailored for the task at hand, that is, the measurement of corpus callosum area changes and its correlation with disease progression. Ishaq also states that the criterion proposed by him is not a new criterion for MSP extraction, rather, it is a novel basis for identification of a plane for measuring corpus callosum area change. For convenience, this minimum corpus callosum area plane will be shortened to "MCAP".

It is important to note that for a single MRI volume the MCAP is not guaranteed to be unique, that is, multiple planes in the brain may have the same minimum corpus callosum area. Since all of these planes restrict the neural transmission equally, identification of one of these planes is sufficient for our purposes.

When searching for the MCAP, one must continuously make use of an image registration implementation. As (spline-based) registration methods are usually computationally intensive, the implemented registration process is accelerated by employing the parallel capabilities of a GPU.

\section{METHOD}

Extracting the MCAP out of an MRI volume is a process that consists out of several stages. The most important stage is image registration, that is used for calculating the cross-sectional area of the corpus callosum. All the necessary steps for finding the MCAP are outlined in the following subsections.

\subsection{Finding the MCAP}

The goal is to extract a plane from an MRI volume which embeds the corpus callosum cross-section with the minimum area; also called the MCAP. This crosssectional area of the corpus callosum will be denoted as $A_{c c}$, and the plane which embeds this minimal area will be denoted as $P_{\text {ext }}$. In other words, this means that the area $A_{c c}$ can be written as a function with $P_{e x t}$ as its parameter:

$$
A_{c c}\left(P_{\text {ext }}\right) \text {. }
$$

This function must be minimized with respect to the parameter $P_{\text {ext }}$. In order to optimize Equation 1, the value of $A_{c c}$ must be calculated, what entails taking the following three steps:

1. Extract a 2-D slice specified by the parameter $P_{\text {ext }}$ from an MRI volume. This entails resampling a plane in the volume. The orientation and position of this plane are parameterized over two rotations $\left(R_{x}, R_{y}\right)$ around the $\mathrm{X}$ - and Y-axes respectively, and one translation $\left(T_{z}\right)$ along the $\mathrm{Z}$-axis. Together, these parameters form the parameter

$$
P_{\text {ext }}=\left(R_{x}, R_{y}, T_{z}\right)
$$

Shackleford reports these variables to take on values between -3.0 and 3.0 (degrees for $R_{x}$ and $R_{y}$ and millimeters for $T_{z}$ ), as the corpus callosum bridge is well defined in this interval. The coordinate system maps the anterior, superior and left directions to the positive $X, Y$, and $Z$ axes respectively and is different from the usually used RAS and LAS coordinate systems.

2. Segment the corpus callosum cross-section in the slice extracted in step 1, by registering a 2-D template with a segmented corpus callosum to the extracted slice;

3. Calculate the area $\left(A_{c c}\right)$ of the corpus callosum by integrating the determinant of the Jacobian of the displacement field over all the points on the template which lie inside the corpus callosum (Davatzikos et al., 1996).

Since the template is a vital part in finding the MCAP, it is manually extracted from the MRI volume using 3D Slicer, which is an open source application.

Ishaq mentions that one can also propose an alternative framework which segments the whole corpus callosum bridge in a given volume, by registering it in 3-D to a pre-segmented template volume and then finding MCAP by slicing the corpus callosum bridge and measuring the corpus callosum area. However, the corpus callosum is mostly a featureless organ. Therefore, such a 3-D registration can potentially cause anatomically different slices from the template and target corpus callosums to map to each other, while this is unlikely to happen in the current solution.

\subsection{Image Registration}

The identification of the MCAP relies heavily on image registration. Image registration is an important 
preprocessing step in medical image analysis. Medical images are used for diagnosis, treatment planning, disease monitoring and image guided surgery and are acquired using a variety of imaging modalities. There are, therefore, potential benefits in improving the way these images are compared and combined. Computerized approaches offer potential benefits, particularly by accurately aligning the information in the different images and providing tools for visualizing the combined images (Hill et al., 2001).

Image registration is a task to reliably estimate the geometric transformation such that two images can be precisely aligned. In this paper, the following terminology will be used: the image that is not changed during the registration process is called the reference or fixed image. The second image that is transformed in such a manner that it increasingly resembles the fixed image, is called the template or moving image.

Any registration technique consists out of these four components:

1. a transformation model, which relates the fixed and moving images;

2. a similarity measure, which measures the similarity between fixed and moving image;

3. an optimization technique, which determines the optimal transformation parameters as a function of the similarity function;

4. a regularization term, which is a technique that evaluates a given candidate deformation and penalizes it if it is implausible.

Each of these components can be implemented in different ways. Over the years, numerous algorithms have been proposed. For more information about some of these algorithms, look to Section 2.

The proposed framework will use sum of squared difference as voxel-based similarity measure, restricting the framework to handle only mono-modality problems. To be more specific, 2-D-2-D mono-modal registration will be performed. A free-form deformation model, using B-splines, will be used to model non-rigid deformations, a diffusion regularizer term will penalize implausible deformations, and steepest descent is the method used for optimization.

In order to improve robustness and speed of the image registration framework, a hierarchical multiresolution approach is adopted. A Gaussian pyramid of both the fixed and moving images will be built that will contain the resampled versions of the images at decreasing resolutions. Starting with the pair of images at the lowest resolution, registration is performed using a coarse grid of control points. The registration results from a previous resolution level are used at the higher resolution level and the registration is run again, stopping only when the full image resolution is reached. Adopting this approach, large deformations can be recovered early at low resolution and more detailed deformations are observed at the increasingly finer resolution levels.

\section{IMPLEMENTATION}

The GPU is an attractive platform to accelerate compute-intensive algorithms (such as image registration) due to its ability to perform many arithmetic operations in parallel. For this paper, the image registration implementation was modeled after the work of Shackleford (Shackleford, 2011; Shackleford et al., 2010; Sharp et al., 2010). Shackleford's efforts resulted in the creation of the Plastimatch framework.

Figure 2 gives an overview of the implemented image registration algorithm. Note that the cost function exists out of two terms: the similarity measure and the regularizer. The total cost function is calculated using a GPU, but only the calculations concerning the similarity measure are explained in this paper, since this is the part where the execution time can be significantly improved.

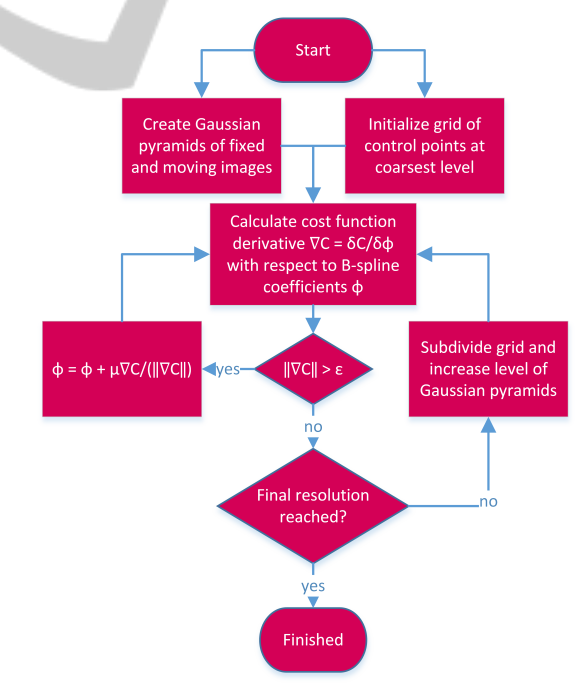

Figure 2: Image registration algorithm outline.

With image registration, a displacement field is calculated that is used to warp the template in such a manner that it resembles the fixed image (in our case, the extracted slice). The displacement field is calculated using the grid of control points and the overall registration process can be viewed as an optimization problem as the similarity measure performed on the two images, has to be minimized. One must also take into consideration the regularization term which will 
penalize implausible deformations. This requires that we evaluate:

1. $C$, the cost function corresponding to a given set of B-spline coefficients;

2. $\partial C / \partial \Phi$, the change in the cost function with respect to the B-spline coefficient values $\Phi$ at each individual control point.

For simplicity, $\partial C / \partial \Phi$ will be called cost function gradient throughout the paper. As mentioned by Shackleford, the B-spline interpolation and the gradient calculation are the two most time-consuming stages within the overall registration process. Therefore, these two stages have been accelerated with CUDA.

In the following subsections, we will first outline the free-form deformation model. Next, we describe how this transformation method is used for generating a displacement field, which will be used for warping the template image. Then, we will describe the implemented data structures and how they are used in calculating the cost function and its derivative. Finally, some final words on the image registration framework's organization.

\subsection{Free-form Deformation Model}

In most image processing applications, the pictures to be manipulated are represented by a set of uniformly spaced sampled values. Unser provided arguments in favor of an approach that uses splines to retrieve these sampled values. They were first described by Schoenberg, where he laid the mathematical foundations for the subject (Schoenberg, 1946). Splines are piecewise polynomials with pieces that are smoothly connected together. Of all the possible polynomial splines, cubic splines tend to be the most popular in applications (Unser, 1999), and are also used in this paper. Cubic B-splines can be represented as follows:

$$
\beta_{l}^{3}(t)=\left\{\begin{array}{l}
\beta_{0}^{3}(t)=(1-t)^{3} / 6 \\
\beta_{1}^{3}(t)=\left(3 t^{3}-6 t^{2}+4\right) / 6 \\
\beta_{2}^{3}(t)=\left(-3 t^{3}+3 t^{2}+3 t+1\right) / 6 \\
\beta_{3}^{3}(t)=t^{3} / 6
\end{array}\right.
$$

The origins of free-form deformation can be traced back to the area of computer aided design (Sederberg and Parry, 1986; Barr, 1984), but it can also be used in medical image analysis (Rueckert et al., 1999). We have chosen for an FFD model based on B-splines.

The basic idea of FFDs is to deform an object by manipulating an underlying mesh of control points. The resulting deformation controls the shape of a 3-D (or 2-D) object and produces a smooth and $C^{2}$ continuous transformation (Rueckert et al., 1999). In

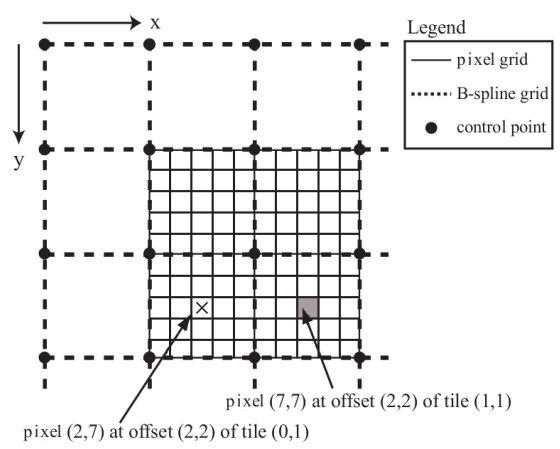

Figure 3: A grid of control points superimposed on the pixels of the template image. Both marked pixels are located at the same relative offset within their respective tiles, so both will use the same $\beta_{l}(u) \beta_{m}(v)$ value.

contrast to thin-plate splines or elastic-body splines, B-splines are locally controlled, which makes them computationally efficient even for a large number of control points. In particular, the basis functions of cubic B-splines have limited support, meaning that changing a certain control point only affects the transformation in the local neighborhood of that control point.

\subsection{Calculating the Displacement Field}

In the case of image registration, the template will be warped in each iteration, until it resembles the fixed image. The FFD transformation model uses a grid of uniformly-spaced control points to calculate the displacement field for the template image, as shown in Figure 3. This results in that the image is partitioned into many, for example, equally sized $5 \times 5$ tiles. Every vector of the displacement field is influenced by the 16 control points in the tile's immediate neighborhood and the B-spline basis function product evaluated at the pixel. The latter is only dependent on the pixel's local coordinates within the tile. For example, in Figure 3 one can see that both marked pixels have the same local coordinates within their respective tiles, namely $(2,2)$. This will result in the same $\mathrm{B}$-spline basis function product value at these two pixels. This property allows the pre-computation of all the relevant B-spline basis function values once, instead of recalculating these values for each individual tile. More on this in the following subsection.

The B-spline interpolation used for the calculation of the X-component of the displacement vector for a certain pixel with coordinates $(x, y)$ is

$$
v_{x}(x, y)=\sum_{m=0}^{3} \sum_{l=0}^{3} \beta_{l}(u) \beta_{m}(v) \phi_{x, i+l, j+m},
$$

where $\phi_{x}$ is the spline coefficient defining the $\mathrm{x}$ - 
component of the displacement vector. The dimensions $n_{x}$ and $n_{y}$ of the control point grid take the following form:

$$
n_{x}=\left\lceil\frac{m_{x}}{s_{x}}+3\right\rceil, \quad n_{y}=\left\lceil\frac{m_{y}}{s_{y}}+3\right\rceil,
$$

where $m_{x}$ and $m_{y}$ are respectively the $X$ and $Y$ dimensions of the template image, and $s_{x}$ and $s_{y}$ are the control point spacing distances. The parameters $i$ and $j$ are the indices of the tile, within which the pixel $(x, y)$ falls, that is

$$
i=\left\lfloor\frac{x}{n_{x}}\right\rfloor-1, \quad j=\left\lfloor\frac{y}{n_{y}}\right\rfloor-1 .
$$

The local coordinates of the pixel within this tile, normalized between $[0,1]$, are

$$
u=\frac{x}{n_{x}}-\left\lfloor\frac{x}{n_{x}}\right\rfloor, \quad v=\frac{y}{n_{y}}-\left\lfloor\frac{y}{n_{y}}\right\rfloor .
$$

\subsection{Optimized Data Structures}

Implementing a data structure that exploits the symmetrical features that emerge as a result of the grid alignment, makes the implementation of Equation 4 much faster. Shackleford considers the following optimizations:

- All pixels within a single tile use the same set of 16 control points to compute their respective displacement vectors. This means that for each tile in the image, the corresponding set of control point indices can be pre-computed and stored in a lookup table (LUT), called the index LUT.

- Equation 7 shows that for a tile of dimensions $n_{w}=n_{x} \times n_{y}$, the number of $\beta(u) \beta(v)$ combinations is limited to $n_{w}$ values. Furthermore, as shown in Figure 3, two pixels belonging to different tiles but with the same local coordinates, will be subject to identical $\beta(u) \beta(v)$ products. This means that a look-up table, called the multiplier LUT, can be calculated containing the precomputed $\beta(u) \beta(v)$ product for all the normalized coordinate combinations.

For each pixel, the absolute coordinates $(x, y)$ within the image dimensions are used to calculate the tile indices that the pixels falls within as well as the pixel's local coordinates within the tile, using Equations 6 and 7 respectively. These tile indices will be used to access the index LUT, which will provide coordinates of the 16 neighboring control points that influence the pixel's interpolation calculation. The pixel's local coordinates within the tile will be used to retrieve the appropriate, pre-calculated $\beta(u) \beta(v)$ product from the multiplier LUT. Using these lookup tables, the displacement field calculation can be considerably optimized.

\subsection{Similarity Cost Function}

Once the displacement field is calculated, it is used to warp the template image. Once warped, the template image is compared to the fixed image (both consisting out of normalized values) by means of the similarity measure. In our case, the similarity measure is the sum of squared differences, that is

$$
C_{\text {sim }}=\frac{1}{N} \sum_{y=0}^{Y} \sum_{x=0}^{X}\left(I_{f}(x, y)-I_{m}\left(x+v_{x}, y+v_{y}\right)\right)^{2},
$$

where $N$ is the amount of pixels in the template image, $X$ and $Y$ are the template image's dimensions, and $\left(v_{x}, v_{y}\right)$ form the displacement field vector for the template image pixel with coordinates $(x, y)$. The similarity part of the cost function will be called the similarity cost function throughout the rest of this paper.

The gradient descent optimization requires the partial derivatives of the similarity cost function with respect to each control point (B-spline) coefficient value. The look-up tables introduced in the previous section not only accelerate the B-spline interpolation stage, it also accelerates the similarity cost function gradient calculation. The similarity cost function gradient can be considered as the change in the similarity cost function with respect to the coefficient values $\Phi$ at each individual control point. This gradient can be decomposed and can be computed independently, for a given control point at the grid coordinates $(\kappa, \lambda)$, as

$$
\frac{\partial C_{\text {sim }}}{\partial \Phi_{(\kappa, \lambda)}}=\frac{1}{N} \sum_{(x, y)}^{16 \text { tiles }} \frac{\partial C}{\partial \vec{v}(x, y)} \frac{\partial \vec{v}(x, y)}{\partial \Phi},
$$

where the summation is performed over all the pixels $(x, y)$ of the template image contained in the 16 tiles found in the control point's local support region. By means of this decomposition, the gradient's dependencies on the similarity cost function and spline coefficients can be independently evaluated. The first term, $\partial C_{\text {sim }} / \partial \vec{v}(x, y)$, depends only on the similarity cost function. The second term, $\partial \vec{v}(x, y) / \partial \Phi$, describes how the displacement field changes with respect to the control points. This last term is only dependent on the B-spline parametrization and the pixel's location; it is computed as

$$
\frac{\partial \vec{v}(x, y)}{\partial \Phi}=\sum_{l=0}^{3} \sum_{m=0}^{3} \beta_{l}^{3}(u) \beta_{m}^{3}(v) .
$$

This only needs to be computed once, since it remains constant over all the optimization iterations. The pre-calculated $\beta(u) \beta(v)$ product is available via the multiplier LUT.

Since the $S S D$ similarity measure is utilized, the first term (see Equation 9) can be written in terms of 
the template image's spatial gradient $\nabla I_{m}(x, y)$ as

$$
\frac{\partial C_{\text {sim }}}{\partial \vec{v}(x, y)}=2 \times\left(I_{f}(x, y)-I_{m}\left(x+v_{x}, y+v_{y}\right)\right) \nabla I_{m}(x, y) \text {. }
$$

Equation 11 shows that it depends on the intensity values of the fixed image and the (warped) template image, $I_{f}$ and $I_{m}$ respectively, as well as the current value of the displacement field $\vec{v}$. Meaning, that during each iteration, the displacement field will change and that results in the modification in the correspondence between the fixed and template images. This also means that, unlike $\partial \vec{v} / \partial \Phi, \partial C_{\text {sim }} / \partial \vec{v}$ needs to be recalculated during each iteration of the optimization. With both terms calculated, they can be combined using the chain rule from Equation 9, which can be written in terms of the control point coordinates $(\kappa, \lambda)$ as

$$
\begin{aligned}
\frac{\partial C_{\text {sim }}}{\partial \Phi_{(\kappa, \lambda)}}=\frac{1}{N} & \sum_{(\kappa, \lambda)}^{16 \text { tiles }} \sum_{b=0}^{s_{y}} \sum_{a=0}^{S_{x}} \frac{\partial C_{s i m}}{\partial \vec{v}(x, y)} \\
& \times \sum_{m=0}^{3} \sum_{l=0}^{3} \beta_{l}^{3}\left(\frac{a}{s_{x}}\right) \beta_{m}^{3}\left(\frac{b}{s_{y}}\right),
\end{aligned}
$$
where $a$ and $b$ are the unnormalized local coordinates of a pixel inside its respective tile, and $x$ and $y$ represent the absolute coordinates of a pixel within the template image. Here, $x$ and $y$ can be defined in terms of the control point coordinates and summation indices as follows:

$$
x=s_{x}(\kappa-l)+a, \quad y=s_{y}(\lambda-m)+b .
$$

For this paper, the method for calculating the similarity cost function gradient has been implemented in three versions: a naïve CPU and GPU version and an optimized GPU version. The next two subsections will describe these implemented versions.

\subsubsection{Naïve CPU/GPU Implementation}

The kernel described by Algorithm 1 calculates the similarity cost function gradient vector $\partial C_{\text {sim }} / \partial \Phi$ for a control point. As previously described, this gradient calculation makes use of the $\partial C_{\text {sim }} / \partial \vec{v}$ and $\partial \vec{v} / \partial \Phi$ terms, as described in Equation 9. The kernel is launched with as many threads as there are control points, where each thread calculates the $\partial C_{\text {sim }} / \partial \Phi$ value for each control point. As shown in the pseudocode, the coordinates $(\kappa, \lambda)$ are deferred from the CUDA thread indices. Each control point's gradient is influenced by 16 neighboring tiles, and the template pixel values that these tiles contain. Once the calculations are finished, the results are stored in the global memory of the GPU.

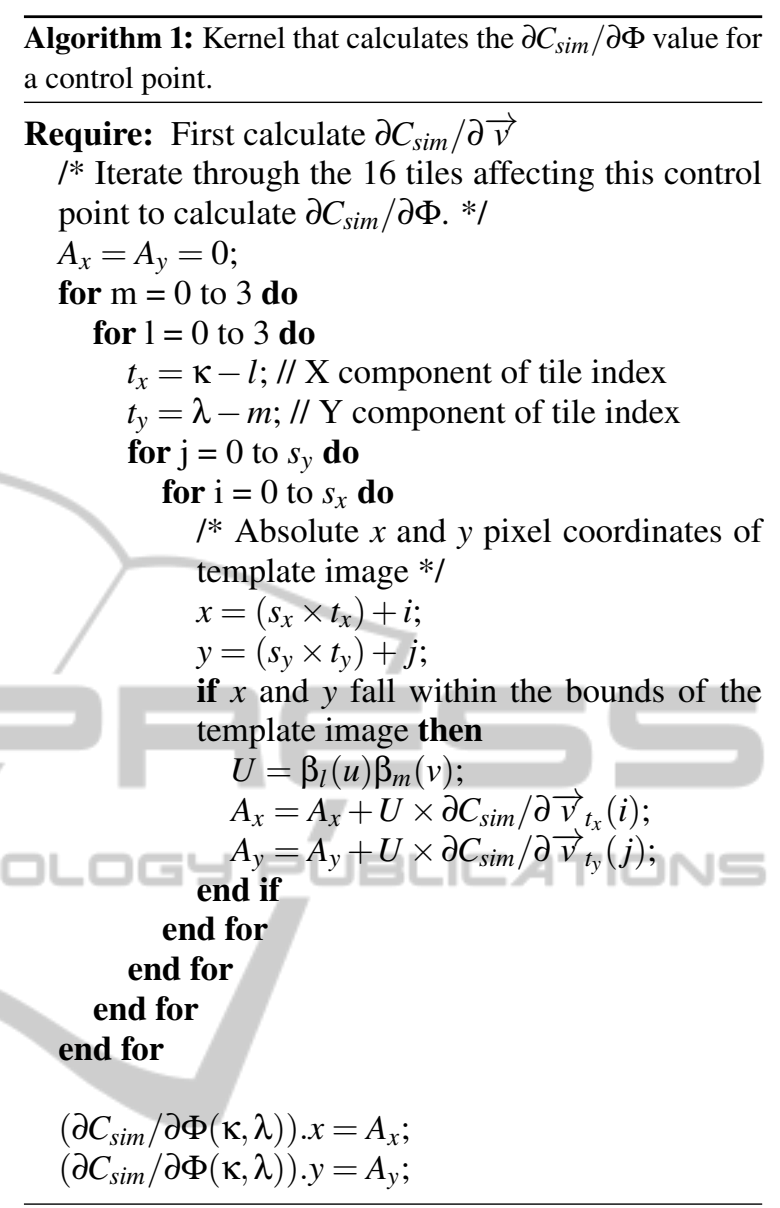

\subsubsection{Optimized GPU Implementation}

Although the implementation described in Section 5.4.1 is a good way to exploit the parallelization capabilities of a GPU, it suffers from serious performance deficiency as the kernel described in Algorithm 1 does a lot of redundant load operations (Shackleford, 2011; Shackleford et al., 2010; Sharp et al., 2010).

As can be seen in Algorithm 1, the gradient value for each control point is influenced by its neighbors. This also implies that when two different threads calculate their respective contribution to a certain one control point, and they both need to calculate the contribution of one the same control point, they must each load the same $\partial C_{\text {sim }} / \partial \vec{v}$ values from the same tile. The only thing these two threads do different is that they must each use different basis-function products when computing the $\partial \vec{v} / \partial \Phi$ term to obtain their respective contributions to the $\partial C_{\text {sim }} / \partial \Phi$ term. To gain a visible view of the problem, consider the following equations. Thread 1 calculates the following contribution of a tile: 


$$
\sum_{s_{x}, s_{y}} \frac{\partial C_{\text {sim }}}{\partial \vec{v}(x, y)} \beta_{0}(u) \beta_{0}(v) .
$$

Thread 2 calculates his contribution of the same tile, but with different $l$ and $m$ values:

$$
\sum_{s_{x}, s_{y}} \frac{\partial C_{\text {sim }}}{\partial \vec{v}(x, y)} \beta_{1}(u) \beta_{2}(v) .
$$

In both Equations 14 and 15, the $u$ and $v$ values are the normalized position of a pixel within the tile. One can clearly see that although these two threads are executed independently of each other in parallel, each thread will end up loading the same $\partial C_{\text {sim }} / \partial \vec{v}$ values, as they are handling the same tile.

This redundant loading of $\partial C_{\text {sim }} / \partial \vec{v}$ values can be mitigated by implementing the following two stages. The first stage will read all the $\partial C_{\text {sim }} / \partial \vec{v}$ values of a certain tile from global memory into shared memory. Any given pixel tile is influenced by (and influences) 16 neighboring pixel tiles, meaning that there are 16 different possible $(l, m)$ combinations. For a certain tile with a certain $(l, m)$ combination, the fol lowing must be calculated

$$
\vec{Z}_{(\kappa, \lambda, l, m)}=\sum_{b=0}^{s_{y}} \sum_{a=0}^{s_{x}} \frac{\partial C_{s i m}}{\partial \vec{v}(x, y, z)} \beta_{l}(u) \beta_{m}(v),
$$

where the values for $x$ and $y$ can be calculated using Equation 13. The operation described in Equation 16 is performed for the 16 possible $(l, m)$ combinations, resulting in $16 \vec{Z}$ values per tile. This operation will be implemented as a GPU kernel. Each of these $\vec{Z}$ values is a partial solution to the gradient computation for a certain control point within the grid. Therefore, we allocate for each control point within the grid an array (or bins) that can hold up to 16 of these partial gradient computation $\vec{Z}$ values. Once the $16 \vec{Z}$ values for a certain tile are computed, each of these values must be inserted into the correct control point's bin of partial gradient computation $\vec{Z}$ values. In other words, when a tile computes the $16 \vec{Z}$ values, these values will not only be written to different control points, but to different bin slots within the control point. The second stage of the gradient computation will simply sum up these $16 \vec{Z}$ values for each control point.

The two proposed stages are implemented as GPU kernels, but only the first stage will be described as the second stage simply sums up all the $16 \vec{Z}$ values of the control point in question. The kernel described in Algorithm 2 will be launched with 16 threads operating on a single tile. Each one of these threads will read a portion of the $\partial C_{\text {sim }} / \partial \vec{v}$ values if this tile into shared memory, so that each of these 16 threads doesn't constantly have to read out of global memory. Once they have been read into shared memory, each thread will compute the $\partial C_{\text {sim }} / \partial \Phi$ value with the appropriate $(l, m)$ values. When a thread finishes this calculation, it puts the computed value into the correct control point's correct bin.

Algorithm 2: Optimized kernel design for calculating the $\partial C_{\text {sim }} / \partial \Phi$ value for a control point. Stage 1 .

Require: First calculate $\partial C_{\text {sim }} / \partial \vec{v}$, get the thread block IDs $(c p X, c p Y)$, get the thread IDs within the thread block $\left(t_{x}, t_{y}\right)$

/* Block IDs */

$c p_{x}=\kappa ; c p_{y}=\lambda$;

/* After reading $\partial C_{\text {sim }} / \partial \vec{v}$ into shared memory is done (left out for brevity) $*$ / _-_synchthreads();

$A_{x}=A_{y}=0$;

for $\mathrm{j}=0$ to $s_{y}$ do

for $\mathrm{i}=0$ to $s_{x}$ do

/* $\mathrm{U}=\beta_{l}(u) \beta_{m}(v) * /$

$A_{x}=A_{x}+U \times \partial C_{\text {sim }} / \partial \vec{v}_{x}(i)$;

$A_{y}=A_{y}+U \times \partial C_{\text {sim }} / \partial \vec{v}_{y}(j)$;

end for

end for

$/ * d c \_d p \_b u c k e t s\left[s_{x} s_{y} \times 16 \times 2\right]$ contains the seperate partial gradient contributions of all the control point's 16 neighbors. $N_{x}$ is the width of the control point grid. */

if $c p_{x}+t_{x}$ and $c p_{y}+t_{y}$ fall within the bounds of the control point grid then

$$
\begin{aligned}
& c p_{t}=c p_{x}+t_{x}+\left(c p_{y}+t_{y}\right) N_{x} ; \\
& \text { bucketID=(3-t } \left.t_{x}\right)+4\left(3-t_{y}\right) ; \\
& \text { dc_dp_buckets }\left[\text { cp } p_{t}\right][b I D][0]=A_{x} ; \\
& \text { dc_dp_buckets }\left[\text { cp } p_{t}\right][\text { bID }][1]=A_{y} ;
\end{aligned}
$$$$
\text { end if }
$$

\subsection{Framework Organization}

The implemented image registration framework uses the GPU for calculations as shown in Figure 4. The $\mathrm{B}$-spline interpolation and the cost function gradient are implemented on the GPU, while the optimization stage is performed on CPU. During each iteration, the optimizer, working on the CPU, calculates new parameters to update the control points so that the cost function is minimized. When a minimum has been found, the registration process stops.

When analyzing Figure 4, one can see that both the evaluated cost function and its gradient must be 


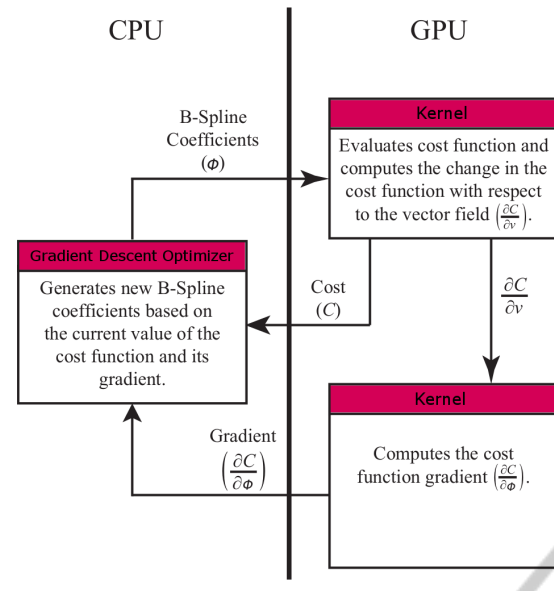

Figure 4: Framework organization.

transferred from GPU to the CPU for every iteration of the image registration process. Transfers between the CPU and GPU memories are the most costly in terms of time, but Shackleford observed in his experiments that the CPU-GPU communication overhead demands roughly $0.14 \%$ if the total algorithm execution time. This fact allows the conclusion that the CPU-GPU transfers do not affect the overall algorithm performance.

\section{RESULTS}

We have implemented two items: an image registration framework and an application that uses this framework for finding the MCAP. Both have been thoroughly evaluated and the results are described in the following subsections.

\subsection{Image Registration Evaluation}

Given that the method for extracting the corpus callosum area relies heavily on deformable registration, the quality of the registration directly affects the accuracy of the area measurement. Several experiments have been conducted in order to evaluate the deformable registration that was implemented. Synthetic data has been used to demonstrate the effectiveness and performance of the registration framework, but our framework has also been evaluated with medical data.

To evaluate the effectiveness and performance of the registration framework, ground truth experiments have been conducted. The precision and error introduced by the algorithm that has to be evaluated, can be assessed by comparing the results with the ground truth. In this case, a synthetic image is deformed using a known control point configuration. This de-

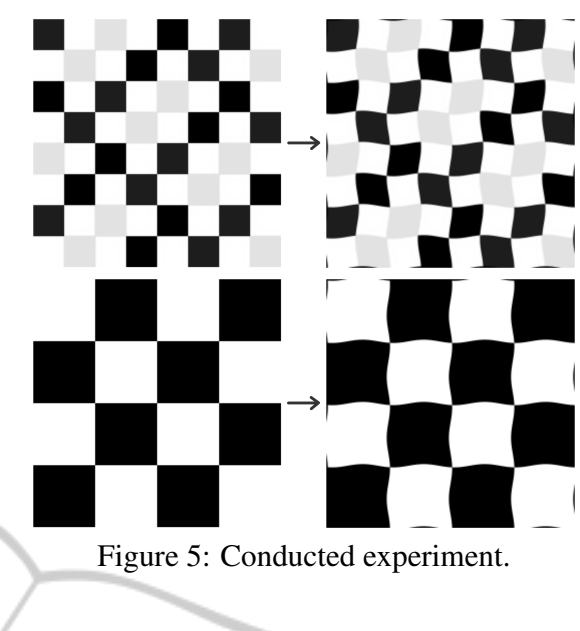

formed image will serve as the reference image, while the original, undeformed image will act as the template image. The quality of the registration can be measured by comparing the displacement field obtained by the registration framework with the ground truth displacement field computed from the known control point configuration.

For the experiments, two synthetic images where used as shown on the left side in Figure 5. Both images have a checkerboard pattern and are of size $256 \times 256$, inspired by the experiments conducted by Schwarz (Schwarz, 2007). For both images, a separate control point configuration was created that were sinusoidal in both $\mathrm{x}$ - and $\mathrm{y}$-direction and with a fixed control point spacing of 15 pixels. Using these control point configurations, the displacement fields were calculated for each original image together with the resulting warped images as shown on the right side of Figure 5. Next, the registration framework is utilized with various control point spacings ranging from 5 to 50 pixels with increments of 5 .

We have conducted three experiments to evaluate the accuracy of our registration framework. First, we calculated the similarity of the images (using SSD) after the registration process (Figure 6). The next experiment calculated the Root Mean Square Error (RMSE) between the ground truth displacement field and the displacement field obtained from the registration process (Figure 7). Last, we calculated the magnitude of difference (Figure 8). Each of these experiments individually validated the accuracy of our registration framework, as they show that a control point spacing of 15-20 pixels best recovers the applied deformation.

For this paper, three versions of the image registration implementation were written: a naïve CPU and GPU implementation and an optimized GPU version. All three implementations will generate the same result, but will perform the registration at dif- 


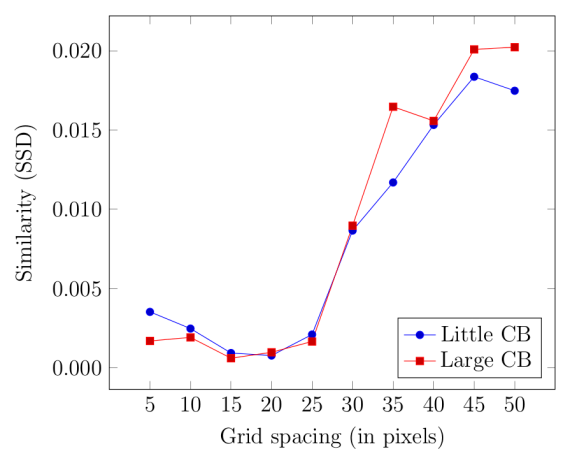

Figure 6: Similarity after registration.

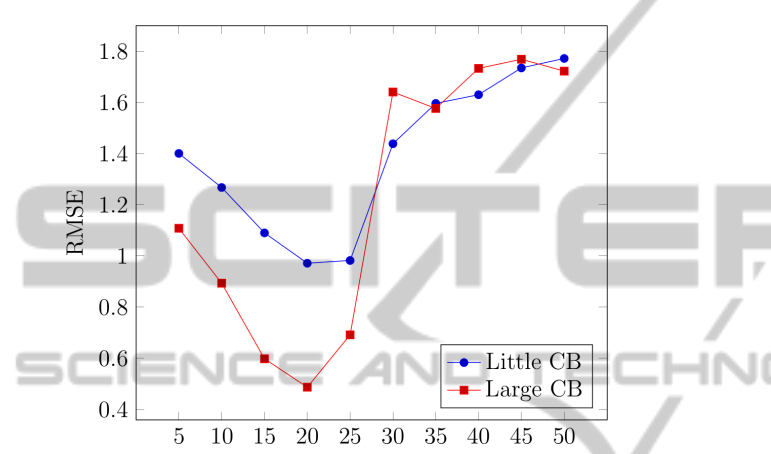

Figure 7: RMSE of the generated displacement field and the ground truth.

ferent speeds. Figure 9 shows the impact of different control point spacings on the three implemented versions. Both the GPU and CPU versions of the naïve implementation are susceptible to grid spacing, because a coarse grid spacing means bigger tiles to compute and a lot of redundant $\partial C_{\text {sim }} / \partial \vec{v}$ loads. The performed experiments showed that when comparing the GPU and CPU version of the naïve implementation, for a grid spacing of 5 pixels, the GPU version performed about 21 times faster than the CPU. When reaching a grid spacing of 50 pixels, it only performed three times faster than the CPU version.

The optimized GPU version, however, is rather agnostic to grid spacing. At a grid spacing of 10 pixels, the optimized version performed about 24 to 40 times faster than the naïve CPU version. When using a grid spacing of 50 pixels, the optimized version calculated its results about 20 times faster than its naïve CPU counterpart. Since the image registration framework is an essential part in finding the MCAP, it is paramount that this part was properly optimized.

The deformable registration framework has also been evaluated using medical data. To assess the quality of the registration, two measurements were used, namely the sum of squared differences (SSD) measurement and the correlation coefficient (CC) measurement, as shown by Figures 10 and 11 respec-

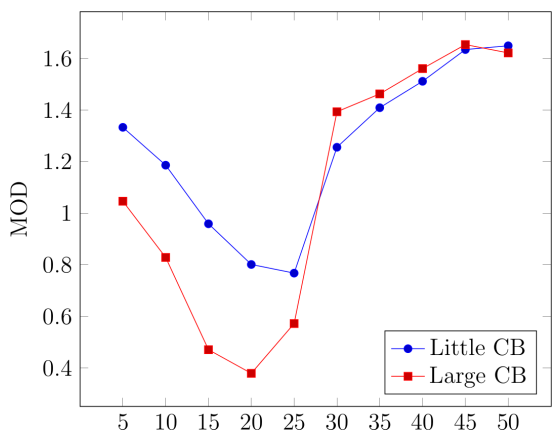

Figure 8: Magnitude of Difference of generated displacement field and ground truth.

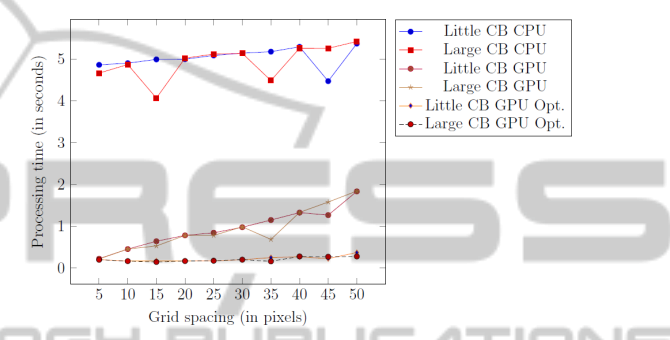

Figure 9: Processing time of registration using CPU, GPU and the optimized GPU versions.

tively. The results show that the resulting warped images significantly resemble their fixed image counterparts. We have used three MRI data sets retrieved from the Open Access Series of Imaging Studies (OASIS) project as well as an MRI volume obtained from icoMetrix. The OASIS project is aimed at making MRI data sets of the brain available to the scientific community. The volume of icoMetrix has a size of $79 \times 95 \times 68$ voxels, while the three volumes from OASIS have a size of $128 \times 256 \times 256$ voxels. However, the three volumes from OASIS are anisotropic in nature. Each of the volumes have been resampled so that they are isotropic, which results in a volume of size $160 \times 256 \times 256$. For each of these volumes, we extracted a plane that roughly resembles the midsagittal plane that would serve as the fixed image. Next, we also extracted from each volume a plane that has been rotated over several degrees from the central pixel of each volume's extracted midsagittal plane. The reason why we chose these planes is that

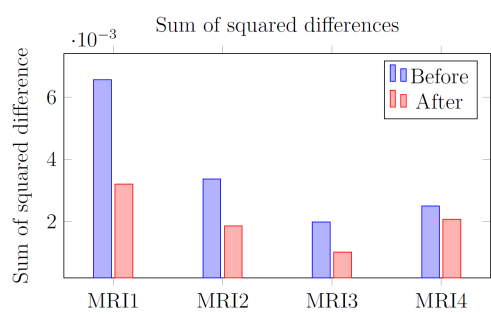

Figure 10: SSD between the fixed image and the warped moving image before and after the registration process. 


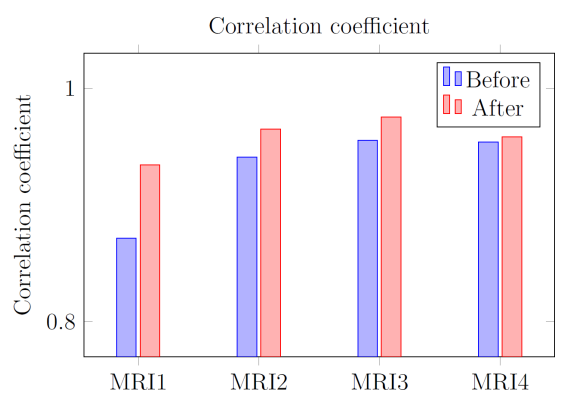

Figure 11: $\mathrm{CC}$ between the fixed image and the warped moving image before and after the registration process.

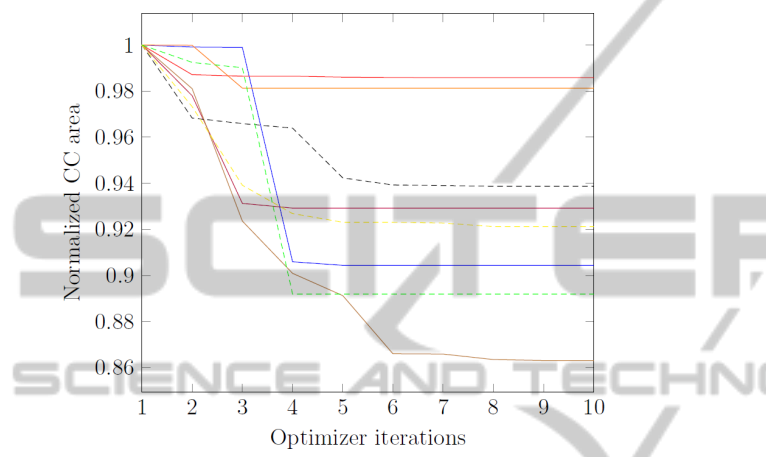

Figure 12: Graph of normalized CC areas of 8 MRI volumes.

this is exactly what the implemented application will do when searching for the MCAP.

\subsection{MCAP Extraction Evaluation}

Figure 12 shows a graph of normalized corpus callosum areas for 8 MRI volumes in function of the optimizer iterations. Seven of these MRI volumes came from the OASIS project and the other one came from icoMetrix. The results for each volume have been normalized (i.e. starting at 1 ) with the rotation parameters and the translation parameter set to zero. The results show a distinct decrease and convergence of the corpus callosum area for all examined volumes.

For the examined volumes, we register a percentage drop ranged from a minimum of $0.01 \%$, a maximum of $13.6889 \%$ with a mean decrease in area of $6.6094 \%$ and a median decrease of $7.5012 \%$. As stated by Ishaq (Ishaq, 2008) and longitudinal study performed by Juha (Juha et al., 2007), the minimization in corpus callosum area is potentially significant, given that approximately $33 \%$ of the reduction in corpus callosum area can be explained by atrophy.

\section{CONCLUSIONS}

The developed framework can automatically extract the MCAP and simultaneously segment the corpus callosum. This method, introduced by Ishaq, can aid longitudinal studies. The method used, treats the corpus callosum area as a function of the plane extraction parameters and it uses deformable registration to generate a displacement field that can be used for the calculation of the corpus callosum area. The gathered results show a clear decrease and convergence to the plane with minimal corpus callosum area.

The obtained results cannot be compared to other, existing methods, because none of the existing methods try to achieve the same objective, which is the identification of minimal corpus callosum area and therefore, such a comparison would not be meaningful. As stated by Ishaq, this work can benefit future studies on longitudinal analysis of the change in corpus callosum area with the progression of different neurological diseases.

Deformable registration is a crucial part in finding the MCAP. The algorithm for deformable registration has been described in detail. Free-form deformation has been used, which allows to model flexible deformations by means of a limited grid of control points, instead of manipulating each pixel individually as is done in deformable registration methods based on dense deformation fields. A regularization term is also used that will penalize deformations that are implausible.

Finding an MCAP requires multiple image registration iterations. Therefore, the registration framework has been optimized using CUDA. The introduced algorithms, optimizations and data structures reduce the complexity of the B-spline registration process. Highly parallel and scalable designs for computing both the sum of squared differences similarity measure and its derivative with respect to the B-spline parameterization were implemented. The speed and robustness of the image registration process were determined using both synthetic and medical data. The acceleration of the GPU process ranges from 24 to 40 times faster than the naïve CPU implementation, depending on the grid spacing used for the control points.

\section{ACKNOWLEDGEMENTS}

This research has been made possible thanks to the collaboration with icoMetrix in Belgium. 


\section{REFERENCES}

Aylward, S., Jomier, J., Barre, S., Davis, B., and Ibanez, L. (2007). Optimizing ITK's Registration Methods for Multi-processor, Shared-memory Systems. MICCAI Workshop on Open Source and Open Data.

Barr, A. (1984). Global and Local Deformations of Solid Primitives. SIGGRAPH Computer Graphics, 18(3):21-30.

Bookstein, F. and Green, D. (1993). A feature space for derivatives of deformation. Information Processing in Medical Imaging - Lecture Notes in Computer Science, 687:1-16.

Christensen, G., Rabbitt, R., and Miller, M. (1996). Deformable templates using large deformation kinematics. IEEE Transactions on Image Processing, 5(10):1435-1447.

Compston, A. and Coles, A. (2008). Multiple Sclerosis. The Lancet, 372(9648):1502-1517.

Davatzikos, C., Vaillant, M., Resnick, S., Prince, J., Letovsky, S., and Bryan, R. (1996). A Computerized Approach for Morphological Analysis of the Corpus Callosum. Journal of Computer Assisted Tomography, 20(1):88-97.

Duning, T. and Kloska, S. (2005). Dehydration confounds the assessment of brain atrophy. Neurology, 64(3):548-550.

Hill, D., Batchelor, P., Holden, M., and Hawkes, D. (2001). Medical Image Registration. Physics in Medicine and Biology, 46(3):R1-R45.

Hubel, D. (1995). Eye, brain and vision. WH Freeman.

Ishaq, O. (2008). Algorithms for Image Analysis of Corpus Callosum Degeneration for Multiple Sclerosis. Master's thesis, Simon Fraser University.

Juha, M., Leszek, S., Sten, F., Jakob, B., Olof, F., and Maria, K. (2007). Non-age-related Callosal Brain Atrophy in Multiple Sclerosis: A 9-year Longitudinal MRI Study Representing Four Decades of Disease Development. Journal of Neurology, Neurosurgery, and Psychiatry, 78:375-380.

Metaxas, D. (1997). Physics-Based Deformable Models: Applications to Computer Vision, Graphics and Medical Imaging. Kluwer.

Rohde, G., Aldroubi, A., and Dawant, B. (2003). The adaptive bases algorithm for intensity-based nonrigid image registration. IEEE Transactions on Medical Imaging, 22(11):1470-1479.

Rueckert, D., Sonoda, L., Hayes, C., Hill, D., Leach, M., and Hawkes, D. (1999). Nonrigid Registration Using Free-Form Deformations: Application to Breast MR Images. IEEE Transactions on Medical Imaging, 18(8).

Schoenberg, I. (1946). Contributions to the Problem of Approximation of Equidistant Data by Analytic Functions. The Quarterly of Applied Mathematics, 4:4599.

Schwarz, L. (2007). Non-rigid Registration Using Freefrom Deformations. Master's thesis, Technische Universität München.
Sederberg, T. and Parry, S. (1986). Free-form Deformation of Solid Geometric Models. SIGGRAPH Computer Graphics, 20(4):151-160.

Shackleford, J. (2011). High-Performance Image Registration Algorithms for Multi-Core Processors. PhD thesis, Drexel University.

Shackleford, J., Kandasamy, N., and Sharp, G. (2010). On developing B-spline registration algorithms for multicore processors. Physics in Medicine and Biology, 55:6329-6351.

Sharp, G., Peroni, M., Li, R., Shackleford, J., and Kandasamy, N. (2010). Evaluation of Plastimatch BSpline Registration on the EMPIRE10 Data Set. Medical Image Analysis for the Clinic: A Grand Challenge, pages 99-108.

Simon, J. (2006). Brain Atrophy in Multiple Sclerosis: What We Know and Would Like to Know. Multiple Sclerosis, 12(6):679-687.

Simon, J., Simon, L., Campion, M., Rudick, R., Cookfair, D., Herndon, R., Richert, J., Salazar, A., Fischer, J., Goodkin, D., Simonian, N., Lajaunie, M., Miller, D., Wende, K., Martens-Davidson, A., Kinkel, R., Munschauer, F., and Brownscheidle, C. (2006). A Longitudinal Study of Brain Atrophy in Relapsing Multiple Sclerosis. Neurology, 12(6):679-687.

Szeliski, R. and Coughlan, J. (1997). Spline-Based Image Registration. International Journal of Computer Vision, 22(3):199-218.

Thirion, J. (1998). Image matching as a diffusion process: an analogy with Maxwell's demons. Medical Imaging Analysis, 2(3):243-260.

Thompson, P. and Toga, A. (1996). A surface-based technique for warping three-dimensional images of the brain. IEEE Transactions on Medical Imaging, 15(4):402-417.

Unser, M. (1999). Splines: A Perfect Fit for Signal and Image Processing. IEEE Signal Processing Magazine, $16(6): 22-38$. 